\title{
Expertise in person recognition
}

\author{
RAYMOND BRUYER and GENEVIÈVE CRISPEELS \\ Louvain University, Louvain-la-Neuve, Belgium
}

\author{
(Claude Bonnet, Sponsor)
}

\begin{abstract}
The dramatic effect of inversion on the episodic recognition of unfamiliar faces has been considered as proof of specific mechanisms for the processing of faces. This view was challenged by Diamond and Carey (1986), who observed similar effects when photographs of dogs were shown to dog experts. However, they compared experts with younger novices, they used identical photographs at encoding and at test, their experts were no more accurate than the novices in the recognition of dogs, and dog recognition is remote from other inputs to the person recognition system such as natural handwriting. In Experiment 1, the results of Diamond and Carey were replicated with natural handwriting as stimuli, with novices matched in age to the experts, and with different structural representations of the targets at encoding and during the recognition test. In Experiment 2, younger novices participated, but this did not affect the pattern of results. Experiment 3 showed that the results were not due to floor effects.
\end{abstract}

The episodic recognition of unfamiliar faces is strongly affected by the upside-down mode of presentation (Yin, 1969; Valentine, 1988, 1991). Classically, the inversion effect (IE) was thought to result from difficulties in the detection of expressive features of the face. However, the recognition of facial expressions and the identification of faces are differentially affected by rotation (Valentine \& Bruce, 1988), and they appear to be processed independently (Young \& Bruce, 1991). Therefore, the IE is now conceived as resulting from difficulties in processing the second-order spatial relationships between facial features (Sergent, 1984; Young, Hellawell, \& Hay, 1987).

First, however, no direct demonstration of qualitatively different mechanisms for upright and inverted faces has ever been reported. Second, Diamond and Carey (1986; D\&C) have challenged the classical view by showing a similar IE for another kind of material, provided that the subjects were experts in the recognition of this type of stimuli. They confirmed the IE for human faces in comparison with photographs of dogs, but the effect for dogs was also observed in the subjects who were dog experts. Thus, the IE cannot be considered as specific to faces. On the contrary, it would apply to any material for which a particular expertise has been acquired. The difference between faces and other materials would be that virtually all normal adults are experts at face recognition. Clinical reports of brain-injured subjects suffering from defective face recognition together with a defect in recognizing exemplars of stimuli for which they were expert before the onset of the brain damage support this view (Bornstein, 1963; Bornstein, Sroka, \& Munitz, 1969; cf., however, Bruyer et al., 1983). It has also been shown that the IE for faces increases from childhood to adulthood (Carey \& Diamond, 1977)-that is, it increases with the development of this expertise.

Correspondence should be addressed to Raymond Bruyer, UCLNECO, Voie du Roman Pays 20, B-1348 Louvain-la-Neuve, Belgium.
The theoretical implications of the observations of D\&C are important. However, they compared the recognition of human faces with the recognition of dogs, and the argument would become stronger if the domain could be restricted to the recognition of persons. First, humans acquire an expertise for that subset of faces to which they are often exposed and from which a prototype seems to be extracted (Goldstein \& Chance, 1980; Valentine, 1991). It would be important to show that the IE is limited to these well-known faces. In support of this idea, Rhodes, Tan, Brake, and Taylor (1989; but cf. Valentine \& Bruce, 1986) observed an IE that was more important for own-race than for other-race faces and Valentine (1991) showed that the IE was more important for own- than for other-race faces and more important for typical than for distinctive faces. Second, the processing of faces is only one way of ensuring the recognition of persons (Young, Hay, \& Ellis, 1985). People are also recognized by their silhouettes, gait, handwriting, voice, and so on, and, outside of laboratories, faces are rarely encountered in isolation.

The present experiments were designed for two purposes. First, we sought to determine whether the IE appears for another input to the person recognition process-natural handwriting. Second, we examined the "expertise" interpretation of the IE by enlisting the participation of both novice and expert subjects. An experimental design identical to that of D\&C's Experiments 2 and 3 was used, with a better control of age differences between novices and experts (D\&C compared undergraduate novices with older experts). Moreover, the "experts" enrolled by D\&C were no more accurate than the novices in the recognition of (upright) dogs. Finally, it is generally assumed that real face (or dog, or handwriting) recognition implies the extraction of invariant features of the stimulus and, therefore, should not be confused with the recognition of a stimulus (Hay \& Young, 1982). Thus, real recognition requires that the encoded representation differ structurally from the representation displayed during the recognition stage. Unfortunately, in the experiments of D\&C, the representa- 
tion provided in the recognition stage was an exact duplicate of the studied picture.

\section{EXPERIMENT 1}

\section{Method}

Stimuli. For faces, two black-and-white photographs of 144 young adult male volunteers, without spectacles or other accessories, were used $(9 \times 13 \mathrm{~cm})$. The two photographs differed in pose and/or expression, and one was involved in the inspection stage while the other was used in the recognition stage. The faces were distributed into targets versus distractors, and upright versus upside-down faces. To ensure equivalent series, 72 pairs of different faces were arbitrarily composed and four independent judges rated the similarity between the two members by means of a 7-point scale. The 72 mean values were then ranked and distributed in the series so that the within-series similarity did not vary. For handwriting, 72 unselected people were asked to copy by hand two short texts. Then, a $5 \times 10 \mathrm{~cm}$ window was cut out and centered for each sample of handwriting. The two texts were equally and randomly distributed as targets or distractors. Similarity judgments and the distribution of the stimuli were made as for faces. In addition, the similarity of handwriting between the two stimuli produced by each writer was evaluated, and the material was distributed accordingly (this "withinwriter" similarity was high: $M=6.36, S D=0.54$ ).

Subjects. Sixteen experts in handwriting (8 females) were recruited from telephone directories. The mean age was 53.4 years $(32-72)$. They had received an education in the domain (graphology) and then practiced a professional activity devoted to the detection of forgeries (for legal affairs). Thirty-two novices (16 females) were recruited from the general public; both samples were comparable in age and sociocultural level. The mean age of novices was 53.3 years (31-70). This group was randomly divided into two groups of 16 subjects each (8 females). One group (53.6 years) participated in the easy handwriting condition, and the other (53.1 years) in the difficult condition ("novices-small" and "novices-large"' subgroups; see below). The three groups did not differ in age $(F<1)$.

Design. Each subject participated in four experimental conditions, the order of which was balanced across the subjects according to a Latin square design. Each condition consisted of an inspection stage, followed by a recognition stage. The recognition of the inspected targets had to be made from a series of pairs of stimuli, consisting of one target and one distractor, according to a forced-choice procedure. The number of pairs in the recognition stage was identical to the number of targets at inspection. The two members of each pair were randomly located one above or below the other. The targets of half the subjects served as distractors for the remaining half. With the exception of targets that appeared both in the inspection and in the recognition stage, every stimulus was seen only once by each subject.

The four conditions were upright faces, inverted faces, upright handwriting, and inverted handwriting. The same orientation was used for the inspection and the recognition stages. For faces, all subjects were presented with a series of 36 targets and then had to recognize them in a series of 36 pairs, each one formed with an old and a new stimulus. For handwriting, the experts and the "novices-large" group received 18 targets and 18 pairs, whereas the "novices-small" group received 9 targets
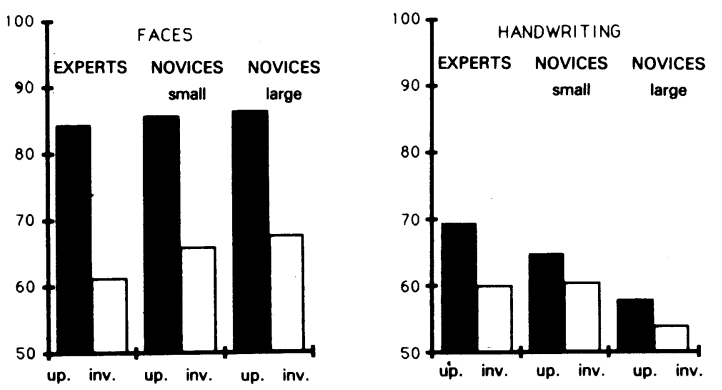

Figure 1. Mean percentage of correct responses as a function of the group, the material, and the orientation in Experiment 1. and 9 pairs. This was done to equalize the accuracy of experts and novices in the upright-handwriting condition, a procedure used by D\&C.

Procedure. During the inspection stage, the targets were shown one by one, for $5 \mathrm{sec}$ each. The subject was invited to inspect them carefully to be able to recognize them a few minutes later. The recognition stage followed immediately. Pairs of stimuli were shown one by one, and the subject, without any time pressure, had to indicate the one inspected previously. Responses were mandatory.

\section{Results}

Figure 1 displays the percentages of correct responses. The results are very similar to those from D\&C. The three subgroups had a large IE with faces, and the experts had the largest IE with handwriting.

The proportions of correct responses were studied by means of a $3 \times 2 \times 2$ analysis of variance (ANOVA). As in $\mathrm{D} \& \mathrm{C}$, the between-subject factor was the group and the within-subject factors were the material and the orientation. Post hoc analyses of the significant effects were computed with the Newman-Keuls test $(p<.05)$. The main group effect was not significant $[F(2,45)=1.23]$, but significant main effects emerged for orientation $[F(1,45)=66.35, p<$ $.0001]$ and material $[F(1,45)=65.96, p<.0001]$. Upright stimuli were recognized better than inverted stimuli, and faces better than handwriting. The material $\times$ orientation interaction was, however, significant $[F(1,45)=$ $15.115, p<.0005]$. While the material effect was significant for both orientations and the IE was significant in both kinds of material, the interaction resulted from a higher IE for faces than for handwriting, and a higher material effect for upright than for inverted stimuli. The significant group $\times$ material interaction $[F(2,45)=4.98, p<.02]$ showed a significant material effect in each group and a significant group effect for handwriting (favoring experts and the novices-small group over the novices-large group) but not for faces. Thus, the material effect was the lowest for experts, and the novices-small subjects processed handwriting as well as did the experts, while the three groups did not differ for faces. However, as in Experiment 2 of D\&C, the three-way interaction was not significant $(F<1)$, despite the suggestion in Figure 1 that experts showed a higher IE than did novices as far as handwriting was concerned.

The crucial feature of the present results is the apparent IE for handwriting in novices. An individual index was computed as (upright - inverted)/(upright + inverted). Such an index weights the difference of performance between upright and inverted material by the general accuracy of the subject. For faces, the index differed significantly from zero for experts $[t(15)=5.52, p<.0001]$, for novices-large $[t(15)=7.17, p<.0001]$, and for novicessmall $[t(15)=5.51, p<.0001]$. For handwriting, the index differed significantly from zero for experts only $[t(15)=2.62, p<.02]$. Thus, when the performance is weighted by the general accuracy of the subject, the apparent IE for handwriting disappeared for novices but remained for experts (and, for faces, in the three samples).

\section{Discussion}

These results are very similar to those of D\&C's Experiment 2. Although D\&C demonstrated the expected interaction in a third experiment in which they increased the required level of expertise, this was 
unnecessary here, since the expected pattern of results was obtained by testing the IE index against zero (D\&C observed the expected pattern in their Experiment 2, but only through the post hoc analysis of a nonsignificant interaction). This similarity of results is interesting because another kind of material was used, and because both the matching of the groups and the preparation of stimuli were improved. In addition, the present results, unlike those of D\&C, confirmed the expected advantage of experts over novices in the recognition of handwriting.

D\&C enrolled young novices and older experts, whereas we recruited novices and experts similar in age; the novices of the present study were older than those studied by D\&C, and the slight discrepancies between the studies could have resulted from this difference. This point was tested in Experiment 2.

\section{EXPERIMENT 2}

\section{Method}

The experiment was identical to Experiment 1, with two exceptionsonly novice subjects were tested, and they were younger than those in Experiment 1 . Thirty-two students were recruited ( 16 females) and randomly assigned to the "large"' (8 females) and "small" groups (8 females). Their mean age was 22.22 years $(19-27)$.

\section{Results}

Figure 2 displays the percentages of correct responses.

The proportions of correct responses were analyzed by means of a 2 (group) $\times 2$ (material) $\times 2$ (orientation) ANOVA. The significant main effect of material $[F(1,30)$ $=183.9, p<.0001]$ revealed that faces were recognized better than handwriting, and the significant main effect of orientation $[F(1,30)=46.49, p<.0001]$ showed the expected IE. There was only one significant interaction that involved precisely these two factors $[F(1,30)=$ $20.79, p<.005]$. The post hoc analysis showed that all differences were significant; that is, the IE was observed for both faces and handwriting and the material effect applied to both the upright and the inverted conditions. Thus, the two main effects were exactly replicated, but the interaction reached a significant statistical level because the IE was higher for faces than for handwriting.

The IE was sound for faces; it appeared in the five groups in both experiments. For handwriting, an additional $3 \times 2$ ANOVA was computed. The between-subject factor was the group, with each of the two groups of Experiment 2 compared with the experts of Experiment 1, and the within-subject factor was orientation. The sole significant effect was the main $\operatorname{IE}[F(1,45)=8.317, p<.006]$.

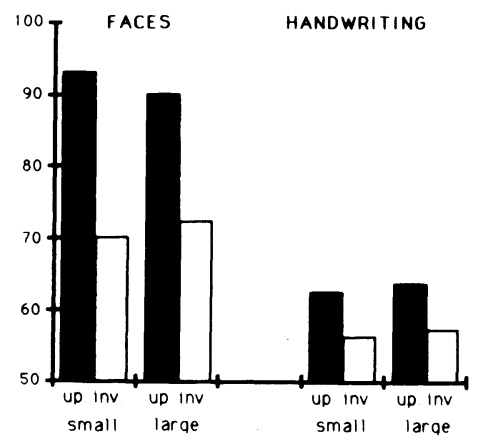

Figure 2. Mean percentage of correct responses as a function of the group, the material, and the orientation in Experiment 2.
Individual IE indexes were derived from the scores of Experiment 2 , and the resulting data were studied by means of a 2 (group) $\times 2$ (material) ANOVA. The sole significant effect was a main advantage of faces over handwriting $[F(1,30)=8.44, p<.007]$. The mean index differed significantly from zero for faces $[t(31)=11.69, p<.0001]$ but not for handwriting.

\section{Discussion}

The scores of younger and older novices were not statistically compared, but it seems obvious that the younger performed better than the older, at least for faces and, to a lesser extent, for handwriting when the large groups are considered. This age effect on episodic memory tasks is largely documented (Kausler, 1985). The main purpose of Experiment 2 was to test whether the effects observed in Experiment 1 could be replicated by enrolling novices of an age similar to that of those studied by D\&C. A positive answer can be given.

The display of an IE for handwriting in experts but not in novices was, admittedly, a bit difficult. The recognition of handwriting was not easy, even in the upright presentation, so that, for novices, there was less room to show a detrimental effect of inversion in the processing of handwriting than there was to show an impairment for faces. Therefore, a third experiment was performed, in which task variables were manipulated to equate performance on upright faces and handwriting and to improve the sensitivity of the results to the inversion.

\section{EXPERIMENT 3}

The basic experiment was modified so that the recognition of faces was expected to become less easy: the exposure duration was reduced in the inspection stage, and a filled interval was provided between the inspection and the recognition. The recognition of handwriting, on the other hand, was expected to become more easy: the exposure duration was increased in the inspection stage, and only the "easy" condition was used (i.e., the "novices-small" condition; in Experiment 1, these subjects did not differ from the experts in the processing of upright handwriting and their performance was less close to the floor effect than was that of the "novices-large" subjects).

For two considerations, Experiment 3 was done with novice subjects. First, a significant IE was found in experts in the recognition of handwriting. Therefore, by increasing the sensitivity of the experimental design, one could only expect a confirmation or an increase of this effect. In addition, the experts were the less susceptible to risk floor effects in the recognition of upright handwriting. The situation was more critical with respect to the novices, because the IE did not appear for them. Thus, by equating the difficulty for upright faces and handwriting and by reaching a sensitive level of performance, one gave rise to the emergence of a significant IE for both faces and handwriting. Second, it appeared impossible to recruit a sample of 16 new experts. As in Experiment 1, middle-aged novices were chosen in lieu of young subjects, to avoid the probable opposite effects of age and expertise observed in Experiment 2.

\section{Method}

The method of Experiment 3 was identical to that of Experiment 1, with three modifications. First, the subjects were novices only. The group was formed with 16 new middle-aged subjects (8 females) (52.3 years; range, 40-65), enrolled as in Experiment 1 . Second, the recognition of faces was hoped to be less easy because of two adaptations. The in- 


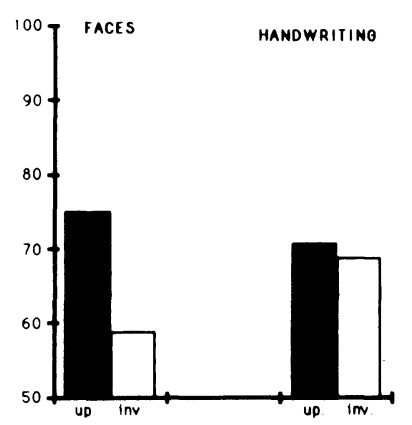

Figure 3. Mean percentage of correct responses as a function of the material and the orientation in Experiment 3.

spection of each target lasted $3 \mathrm{sec}$ instead of $5 \mathrm{sec}$, and a filled interval (counting backward) of 2 min was inserted between the encoding stage and the recognition stage. Third, we hoped to increase the ease of recognition of handwriting by providing an inspection time of $10 \mathrm{sec}$ instead of $5 \mathrm{sec}$, and by using the "novices-small" condition only.

\section{Results}

The percentages of correct responses are displayed in Figure 3. The proportions of correct responses were analyzed by means of a $2 \times 2$ ANOVA. There was a significant main advantage of the upright over the inverted presentation $[F(1,15)=41.65, p<.0001]$. The main effect of material did not reach a significant threshold. However, the material $\times$ orientation interaction was statistically significant $[F(1,15)=38.7, p<.0001]$. The post hoc analysis showed that performance was significantly worse in the inverted-face condition than in the three remaining conditions, which did not differ significantly from each other.

IE indexes were compared to determine any possible effect of the material. The Student $t$ test proved to be statistically significant $[t(15)=6.71, p<.0001]$-the index was significantly higher for faces than for handwriting. The index for faces differed significantly from zero $[t(15)=18.03, p<.0001]$, but the index for handwriting did not differ significantly from zero.

\section{Discussion}

The recognition of upright faces decreased from $86 \%$ to $75 \%$, the recognition of upright handwriting increased from $65 \%$ to $71 \%$, and there was no effect of the material on performance in the upright condition. In addition, the general accuracy ( $73 \%$ ) was reasonably far from both floor and ceiling effects.

And yet, the previous conclusions can be maintained and the pattern of results is even clearer than before. Indeed, the material $x$ orientation interaction showed a significant IE for faces but not for handwriting, the index for faces was significantly higher than for handwriting, and the index for faces differed significantly from zero while the index for handwriting did not differ from zero.

\section{GENERAL DISCUSSION}

The recognition of handwriting was better for experts than for novices in the first, but not the second, experiment. This confirms their expertise and suggests that a detrimental effect of age can cross the favorable effect of expertise. This probably occurred in the study of D\&C.

D\&C challenged the classical view, according to which the IE on the recognition of unfamiliar faces reveals mechanisms specific to the processing of normally oriented faces. Indeed, these authors observed a similar effect when photographs of dogs were shown to dog experts. They concluded that this phenomenon involved visuospatial skills for which a particular expertise had been acquired, rather than face-specific processes.
The present study replicated these observations. The IE was observed for faces in experts and novices, and for handwriting in experts only. The present study generalizes the observations of D\&C in three major directions, but limits them in two ways. First, D\&C's results were probably not biased by the age differences between their experts and their novices, since the same pattern appeared in the present study with both young and older novices. Second, the effects were replicated while a true recognition of faces and handwriting was ensured, since the studied material differed structurally from the recognized material (this probably explains why the subjects of the present study were slightly less accurate than those in D\&C). This point constitutes the first limitation of their research. Third, the effects were replicated with the use of natural handwriting, a type of material that is more related to face recognition than are photographs of dogs. However, given the first limitation of the D\&C study, this last point leads us to the second limitation. Indeed, a doubt remains as to whether the IE has been observed in the true recognition of dogs by dog experts.

The lesson of these two studies is that the IE is not limited to faces, for it can be generalized to at least one other visual input to the person recognition system, handwriting. It remains to be seen whether the expertise interpretation is person specific.

\section{REFERENCES}

Bornstein, B. (1963). Prosopagnosia. In L. Halpern (Ed.), Problems of dynamic neurology (pp. 283-318). Jerusalem: Hadassah Medical Organization.

Bornstein, B., SRokA, H., \& Munitz, H. (1969). Prosopagnosia with animal face agnosia. Cortex, 5, 164-169.

Bruyer, R., Laterre, C., Seron, X., Feyereisen, P., StrypStein, E., Pierrard, E., \&ectem, D. (1983). A case of prosopagnosia with some preserved covert remembrance of familiar faces. Brain \& Cognition, 2, 257-284.

Carey, S., \& Diamond, R. (1977). From piecemeal to configurational representation of faces. Science, 195, 312-314.

Diamond, R., \& CAREY, S. (1986). Why faces are and are not special: An effect of expertise. Journal of Experimental Psychology: General, $115,107-117$

Goldstein, A. G., \& Chance, J. E. (1980). Memory for faces and schema theory. Journal of Psychology, 105, 47-59.

HAY, D. C., \&oung, A. W. (1982). The human face. In A. W.Ellis (Ed.), Normality and pathology in cognitive functions (pp. 173-202). New York: Academic Press.

KAUSLER, D. H. (1985). Episodic memory: Memorizing performance. In N. Charness (Ed.), Aging and human performance. (pp. 101-141). Chichester, U.K.: Wiley.

Rhodes, G., TAN, S., BRAKe, S., \& TAYLOR, K. (1989). Expertise and configural coding in face recognition. British Journal of Psychology, 80, 313-331.

SERGENT, J. (1984). An investigation into component and configural processes underlying face recognition. British Journal of Psychology, 75, 221-242.

VAlEntine, T. (1988). Upside-down faces: A review of the effect of inversion upon face recognition. British Journal of Psychology, 79, 471-491.

VALENTINE, T. (1991). A unified account of the effects of distinctiveness, inversion and race in face recognition. Quarterly Journal of Experimental Psychology, 43A, 161-204.

VAlENTINE, T., \& BRUCE, V. (1986). The effect of race, inversion and encoding activity upon face recognition. Acta Psychologica, 61, 259-273.

VAlentine, T., \& BRUCE, V. (1988). Mental rotation of faces. Memory \& Cognition, 16, 556-566.

YIN, R. K. (1969). Looking at upside-down faces. Journal of Experimental Psychology, 81, 141-145.

Young, A. W., Bruce, V. (1991). Perceptual categories and the computation of "Grandmother." European Journal of Cognitive Psychology, 3, 5-49.

Young, A. W., HAY, D. C., \& Ellis, A. W. (1985). The faces that launched a thousand slips: Everyday difficulties and errors in recognizing people. British Journal of Psychology, 76, 495-523.

Young, A. W., Hellawell, D., \& Hay, D. C. (1987). Configural information in face perception. Perception, 16, 747-759.

(Manuscript received March 4, 1992.) 